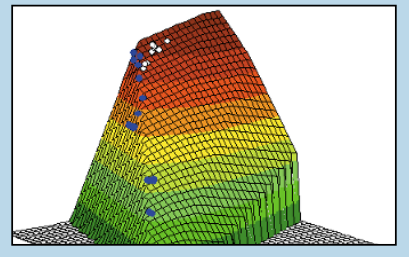

Several approaches exist for the mathematical modeling of cellular metabolism and its regulation, but most of them require detailed kinetic and concentration information about enzymes and various cofactors that is difficult to obtain. Taking a different tack, Bernhard Palsson and colleagues had previously reconstructed an Escherichia coli metabolic network using a stoichiometric, rather than a kinetic approach. The method relies on the application of known constraints on the integrated function of reconstructed networks and does not lead to a single solution but instead provides a domain of possible solutions that represent allowable functions of the network. On page 125, they apply their model to test the hypothesis that $E$. coli uses its metabolism to maximize its growth rate. They found that experimentally determined growth rates and substrate and oxygen uptake rates agreed with the a priori calculated predictions of the model, validating the in silico approach's ability to interpret and predict cellular function (see also p. 111).

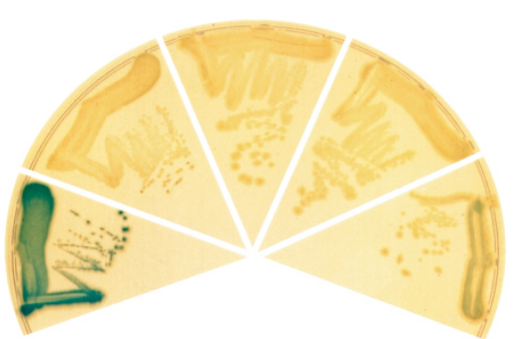

Protein mis-

folding is associated with several human diseases, including Alzheimer's, and can hinder the efficient production of soluble recombinant proteins. In this issue, Wigley et al. (see p. 131) present a method for assessing the solubility and folding of expressed proteins in vivo. The assay is based on the generation of functional $\beta$-galactosidase $(\beta$-gal) in Escherichia coli by complementation of two fragments of the enzyme. By fusing one of the fragments to the $C$ terminus of a target protein, such as $A \beta$, the Alzheimer's amyloid peptide, the generation of functional $\beta$-gal is made dependent on the solubility of the fusion protein. Enzyme activity is monitored by a color change. The assay should be applicable to screening for drugs that promote the folding or inhibit the aggregation of diseaserelated proteins (see also p. 112). MS

\section{Calcium sensor upgrade}

Green fluorescent protein (GFP)-based calcium probes have been developed, but their limited signal intensity has made it difficult to measure calcium concentrations with good spatial and temporal resolution. On page 137 Nakai et al. describe a high-affinity calcium probe based upon a previously described circularly permutated enhanced GFP (cpEGFP) molecule. They engineered the probe by attaching the $\mathrm{N}$ terminus of the cpEGFP molecule to the M13 fragment of myosin light chain kinase-the target sequence of the calcium-binding protein calmodulin-and they then connected the $\mathrm{C}$ terminus to calmodulin. When calcium binds to calmodulin, conformational changes due to the calcium-calmodulin-M13 interaction induce a subsequent confor-

mational change in cpEGFP, so
that the fluorescence intensity
changes. The authors observed
large fluorescence changes in
cells expressing the sensor in
response to application of drugs
or electrical stimulation. The
sensor will be a useful tool for
visualizing calcium in living
cells.

\section{Technical Reports}

On page 167 Harpur et al. present a fluorescence resonance energy transfer (FRET) imaging method that enables the use of bright, but previously incompatible fluorescent protein pairs, such as spectrally bright yellow/green fluorescent proteins (EYFP and EGFP), to measure FRET in individual living cells. They applied the technique to monitor caspase activity in cells during apoptosis. To do this, they inserted a caspase cleavage site between the spectrally similar EYFP/EGFP pair and measured FRET by determining the fluorescence lifetime of the combined donor/acceptor pair by fluorescence lifetime imaging microscopy. Loss of FRET upon cleavage of the site results in shorter fluorescence lifetime.

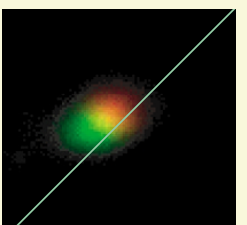

The hormone leptin, produced by fat cells, has been suggested as a potential obesity therapy. However, recent studies suggest that therapeutic approaches that deliver the leptin

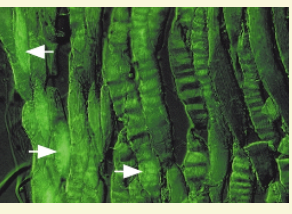
protein systemically may be problematic, indicating the need for targeting of the gene product to specific cells in the central nervous system. With this end in mind, Mulligan and colleagues have delivered leptin to the mouse hypothalamus by intracranial gene transfer using an adeno-associated viral vector, demonstrating efficient body weight control in obese mice (see p. 169).
Next Month in:

\section{Nature Biotechnology}

\author{
Early flowering \\ citrus
}

\section{Nanopore SNP detection}

Identifying essential genes
in Candida

\author{
Engineering \\ microvasculature
}

